\title{
Do Automated Telephone Systems Decrease Hospital Readmissions Among Patients with Cirrhosis?
}

\author{
Bruce Runyon $^{1} \cdot$ Lavinia Mitroi $^{2}$
}

Received: 7 September 2015/ Accepted: 10 September 2015/Published online: 22 September 2015

(c) Springer Science+Business Media New York 2015

Even though the traditional adage "everyone needs a good listening to" is doubly true within the patient-physician relationship, patients often have difficulty reaching their physician once they exit the examination room, with the patient, front desk, and the physician playing "telephone tag" rather than effectively communicating, tragically culminating at times with a visit to the emergency department for patients suffering from more serious conditions.

In assessing core qualities that influence patient satisfaction, communication issues appear to influence low physician ratings, even in the presence of other positive qualities [1]. Furthermore, even though part of the movement toward "patient-centered care" includes timely communication with a physician's office, in practice automated answering systems and message taking contribute to largely negative patient perceptions [2] despite the data supporting the use of technology including automated telephone systems, standard telephone calls, e-mails, and faxes as facilitating the management of multiple chronic conditions [3].

Modern technology has opened multiple channels of communication between patients and their physicians, whether through telephone calls, secure messaging systems, or Bluetooth-enabled technology such as "smart scales," all of which can serve to prevent unnecessary emergency department visits. It is vital, however, to

Lavinia Mitroi

lmitroi@mail.harvard.edu

1 Department of Medicine, University of New Mexico School of Medicine, Albuquerque, NM, USA

2 DrPH Program, Harvard T.H. Chan School of Public Health, Boston, MA, USA consider the preferences and familiarity of the patient population with these technologies prior to implementation of a novel communication method. Elderly patients usually prefer to receive personal assistance from a human operator, whereas younger patients typically feel more comfortable with a "robocall," texting, or e-mail.

In this issue of Digestive Diseases and Sciences, Thomson et al. [4] describe and validate the use of an automated telephone monitoring system with interactive voice response (IVR) in the prediction of readmission for patients with cirrhosis. Despite patient dissatisfaction with automated telephone systems cited by other studies, this pilot study reported a high level of compliance, with $70 \%$ of patients completing over $80 \%$ of their IVR assessments, particularly among patients with cirrhosis, who are frequently noncompliant. Furthermore, the responses provided by this population enabled the authors to successfully predict which subjects would be hospitalized, while increasing the value of face-to-face encounters with the treating physician(s) through the provision of additional information [4].

IVR monitoring has the potential to improve the efficiency and effectiveness of hepatology care by predicting which patients are stable enough to require less frequent communication versus patients who demand additional interventions. Nevertheless, since responses collected from automated telephone systems are largely dichotomous (yes/ no) or ordinal (limited to a Likert scale rating of how the patient is feeling), these types of data are not conducive to immediate intervention. As the authors note, although scheduled calls with a member of the patient's care team between office visits could provide more detailed data for informing management decisions, these calls are resource intensive.

We have some experience with a pilot study utilizing a liver care coordinator (LCC) as a comprehensive point-of- 
Table 1 Comparing the costbenefit of automated phone systems versus care management interventions in preventing readmissions among patients with cirrhosis

\begin{tabular}{ll}
\hline Automated phone systems & Care management interventions \\
\hline High setup cost & Low setup cost \\
Greater communication & Greater communication \\
Increased data for decision-making & Increased data for decision-making \\
Mostly dichotomous, ordinal data & Mixed data \\
No additional services & Increased access to wrap-around services \\
Economies of scale & No economies of scale \\
Both: potential for standardized interventions toward prevention of readmissions
\end{tabular}

contact between patients with cirrhosis and their treating hepatologist. Patients with advanced liver disease called in daily or weekly to report their basic data such as blood pressure, weight, and mental status in order to inform medical management decisions or to recommend additional office visits. Furthermore, the LCC acted as a patient "navigator," connecting them to their PCP, other specialists, or to care team members, assisting with insurance prior to authorizations for medications and screenings, and connecting patients to other resources, as needed. The LCC pilot study, which had a lower initial cost than the IVR system $(<\$ 60,000)$, collected actionable patient data (beyond dichotomous/ordinal responses) and provided a possibility for a rapid intervention (Table 1). As Thomson et al. note, care management interventions such as the LCC model, which involve human intervention, do not benefit from the economies of scale, with expenses increasing over time and with expansion of the patient pool.

As such, the IVR system represents an excellent first step toward improving patient-physician communication. Increasing the amount of information available to hepatologists between face-to-face office visits provides the opportunity for improving care and for reducing preventable and costly rehospitalizations. In the future, the prognostic data collected by the IVR system could be used to formulate standardized interventions.

\section{References}

1. Anderson R, Barbara A, Feldman S. What patients want: a content analysis of key qualities that influence patient satisfaction. $J$ Med Pract Manag. 2007;22:255-261.

2. Berckelaer AV, DiRocco D, Ferguson M, Gray P, Marcus N, Day S. Building a patient-centered medical home: obtaining the patient's voice. JABFM. 2012;25:192-198.

3. Koch G, Wakefield BJ, Wakefield DS. Barriers and facilitators to managing multiple chronic conditions: a systematic literature review. West J Nurs Res. 2015;37:498-516.

4. Thomson M, Volk M, Kim HM, Piette J. An automated telephone monitoring system to identify patients with cirrhosis at risk for rehospitalization. Dig Dis Sci. (Epub ahead of print). doi:10.1007/ s10620-015-3744-3. 